BIOLOGICAL CRYSTALLOGRAPHY

ISSN 1399-0047

Received 27 January 2015

Accepted 9 April 2015

Edited by Z. S. Derewenda, University of Virginia, USA

Keywords: SAXS; HPLC; oligomer; processing.

Supporting information: this article has supporting information at journals.iucr.org/d

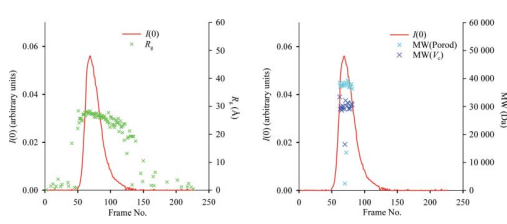

C 2015 International Union of Crystallography

\section{DATASW, a tool for HPLC-SAXS data analysis}

\author{
Alexander V. Shkumatov* and Sergei V. Strelkov
}

Department of Pharmaceutical and Pharmacological Sciences, KU Leuven, Herestraat 49, 3000 Leuven, Belgium.

*Correspondence e-mail: alexander.shkumatov@gmail.com

Small-angle X-ray scattering (SAXS) in solution is a common low-resolution method which can efficiently complement the high-resolution information obtained by crystallography or NMR. Sample monodispersity is key to reliable SAXS data interpretation and model building. Beamline setups with inline highperformance liquid chromatography (HPLC) are particularly useful for accurate profiling of heterogeneous samples. The program $D A T A S W$ performs averaging of individual data frames from HPLC-SAXS experiments using a sliding window of a user-specified size, calculates overall parameters $\left[I(0), R_{\mathrm{g}}, D_{\max }\right.$ and molecular weight] and predicts the folding state (folded/unfolded) of the sample. Applications of DATASW are illustrated for several proteins with various oligomerization behaviours recorded on different beamlines. DATASW binaries for major operating systems can be downloaded from http://datasw.sourceforge.net/.

\section{Introduction}

The application of small-angle X-ray scattering (SAXS) to study the structure and properties of biological macromolecules in solution has seen exponential growth over the last decade (Graewert \& Svergun, 2013). SAXS can provide a rapid assessment of parameters such as molecular weight (MW), radius of gyration $\left(R_{\mathrm{g}}\right)$ and the overall shape of a macromolecule, thereby complementing high-resolution crystallography or nuclear magnetic resonance studies (Elegheert et al., 2012; Lapinaite et al., 2013). SAXS can be particularly useful for the characterization of oligomerizationprone (Soderberg et al., 2013) and flexible (Shkumatov et al., 2011) proteins.

If a solution contains multiple macromolecule species, the measured SAXS curve will be an average of all these species. Deconvolution of such curves is far from trivial (Petoukhov et al., 2012). A more efficient separation can be achieved by passing the sample through an HPLC system while the SAXS signal from the eluate is continuously recorded (Mathew et al., 2004). The existing tools for the analysis of HPLC-SAXS data include automatic data-analysis pipelines that are typically run in real time during data collection, allowing very limited adjustment by the user, and standalone programs such as FOXTROT (David \& Pérez, 2009) and US-SOMO (Brookes et al., 2010). FOXTROT, developed at the SWING beamline, can only perform frame-per-frame buffer subtraction and calculation of $R_{\mathrm{g}}$ and $I(0)$ using fixed data range for all frames specified by the user. US-SOMO is a sophisticated analysis and modelling framework for various experimental data recently supplemented by an HPLC-SAXS module, albeit still under development (Brookes et al., 2013). Overall, US-SOMO can be quite cumbersome for novice SAXS users.

$D A T A S W$ was developed for the rapid analysis of subtracted HPLC-SAXS, calculating for each data frame the 
Table 1

$D A T A S W$ strategy to process subtracted HPLC-SAXS data.

\begin{tabular}{|c|c|c|}
\hline Action & Processing tool & Output file name \\
\hline Frame averaging using user-specified sliding window & $D A T A V E R$ & - \\
\hline $\begin{array}{l}\text { Automatic computation of } R_{\mathrm{g}} \text { and } I(0) \text { using the Guinier } \\
\text { approximation, filtering out low-quality frames based on } R_{\mathrm{g}} \\
\text { quality estimate }\end{array}$ & AUTORG & $\begin{array}{l}\text { autorg_summary.txt; autorg_summary-Peak.txt; } \\
\text { Frame-versus-IORg.txt }\end{array}$ \\
\hline Prediction of folding state (folded/unfolded) & $D A T C L A S S$ & autorg_summary-Peak.txt \\
\hline Peak detection based on $I(0)$ values & Built-in $\dagger$ & \\
\hline $\begin{array}{l}\text { Estimation of } D_{\max } \text {, the distance distribution function } P(r) \\
\text { and the regularized scattering curve }\end{array}$ & $D A T G N O M$ & MW_estimation.txt; Frame-versus-MWs.txt \\
\hline $\begin{array}{l}\text { Computation of Porod volume from the regularized scattering } \\
\text { curve and MW }\end{array}$ & DATPOROD & \\
\hline Estimation of MW from the volume of correlation & $D A T V C$ & \\
\hline $\begin{array}{l}\text { Generation of plots [frame versus } I(0) / R_{\mathrm{g}} \text { and frame versus } \\
\left.\mathrm{MW}(\text { Porod }) / \mathrm{MW}\left(V_{\mathrm{c}}\right)\right]\end{array}$ & GNUPLOT & $\begin{array}{l}\text { frame-versus-iOrgmw pdf or frame-versus-i0rgmw.ps } \\
\quad \text { (Unix) }\end{array}$ \\
\hline
\end{tabular}

$\dagger$ With the exception of these two tools, the tools are from the ATSAS package (Petoukhov et al., 2012).

zero-angle scattering $I(0)$, the radius of gyration $R_{\mathrm{g}}$, the maximal dimension $D_{\max }$ and two estimates of the MW from the Porod volume $V_{\mathrm{p}}$ and the volume of correlation $V_{\mathrm{c}}$ (Rambo \& Tainer, 2013), as well as suggesting the folding state (folded/unfolded). DATASW plots calculated parameters as a function of frame number and a publication-ready figure is generated.

\section{Implementation and requirements}

$D A T A S W$ is written in Perl. The program supports various datafile-naming formats used at SAXS beamlines. DATASW requires tools from the $A T S A S$ (v.2.5.2 or higher) program package (Petoukhov et al., 2012; Table 1). For peak detection, a simple built-in algorithm is used: a baseline is calculated as an average over computed $I(0)$ values for all subtracted frames; thereafter, groups of frames with $I(0)$ values above the mean value are identified as peak areas. MW estimation and peak detection are only performed for frames where $R_{g}$ can be reliably estimated by $A U T O R G$ (Table 1), i.e. the $R_{\mathrm{g}}$ quality estimate must be higher than $10 \%$ (where $0 \%$ corresponds to unusable data and $100 \%$ to data of ideal quality) with a standard deviation of no more than $30 \%$. The calculated Porod volume is divided by 1.7 to obtain an estimate of the molecular weight (Petoukhov et al., 2012). GNUPLOT (http:// www.gnuplot.org) is used to generate a figure containing plots of $R_{\mathrm{g}}, I(0)$ and MW versus frame number.

\section{Features}

An HPLC-SAXS experiment results in a set of files with intensity versus scattering angle recorded as a series of 'frames' with user-defined exposure time. Final frames need to be buffer-subtracted, as performed by automatic pipelines at the BM29 and P12 beamlines or manually by the user: the FOXTROT software is used at the SWING beamline. As an input for $D A T A S W$, one or more folders with subtracted datafiles are used. Typically, buffer-subtracted datafiles should
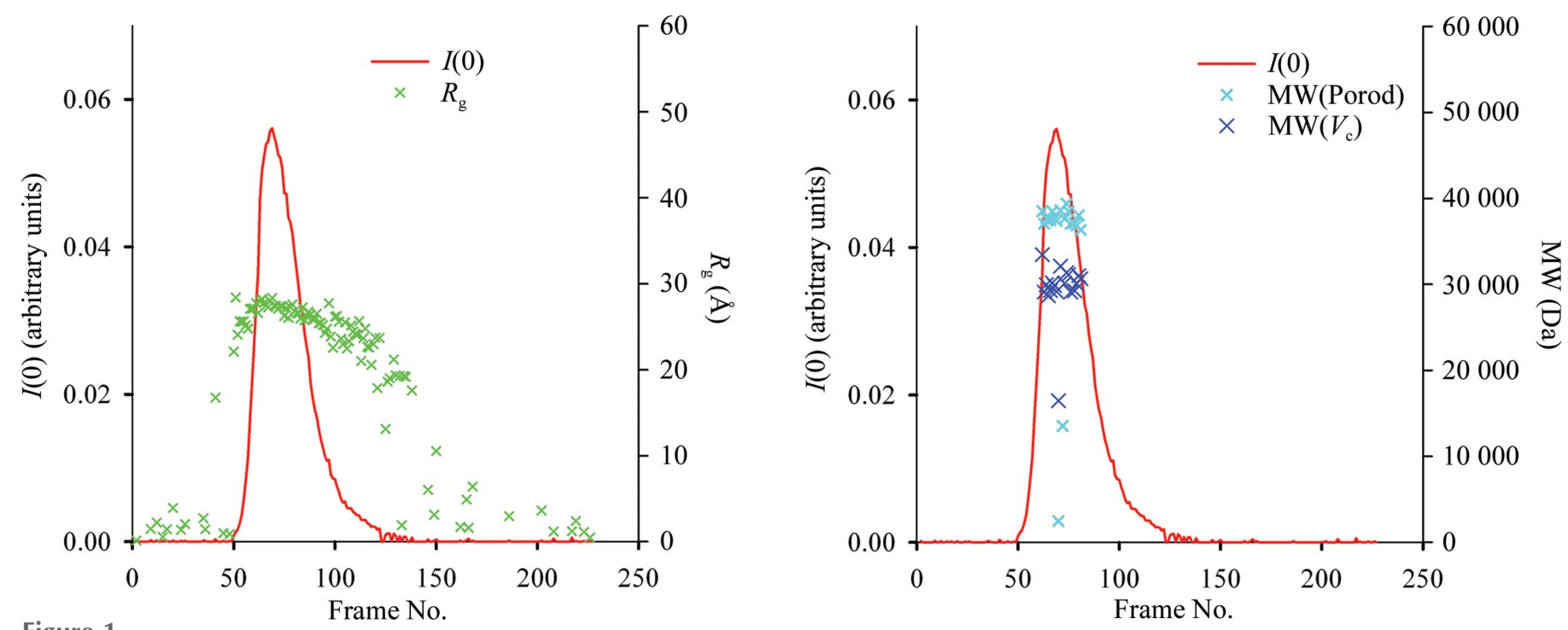

Figure 1

Output generated by DATASW for a typical monodisperse sample (HSPB6 dimer; Weeks et al., 2014). The forward scattering $I(0)$ and radius of gyration $R_{\mathrm{g}}$ are shown as a red line and green crosses, respectively (left). MW determined from $V_{\mathrm{p}}$ (cyan crosses) and $V_{\mathrm{c}}$ (blue crosses) are plotted versus frame number (right). MW is only calculated for the frames of the automatically determined peak. 
have a common identifier optionally followed by some text, an underscore separating a sequential number and a dat extension. It is recommended that the files should be prepended with a common identifier, e.g. 'hplc', to enable the processing of multiple folders. DATASW can be run in interactive or batch mode, and for the latter the most important parameters can be supplied as arguments on the command line. The output includes a folder with subtracted data files, optionally averaged using a sliding window size specified by the user. This step may be necessary to increase the signal-to-noise ratio (SNR) in case the concentration of the injected sample was low. The output folder also contains files and figures reflecting calculated parameters (Fig. 1, Table 1). Peak detection can be disabled for oligomerization-prone

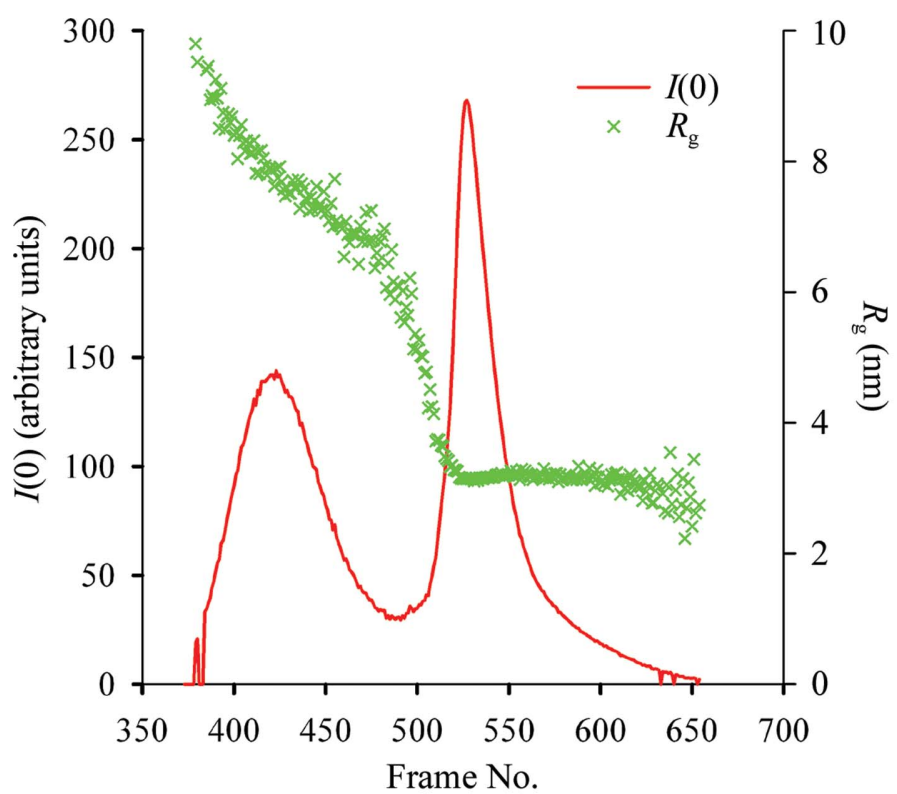

(a)

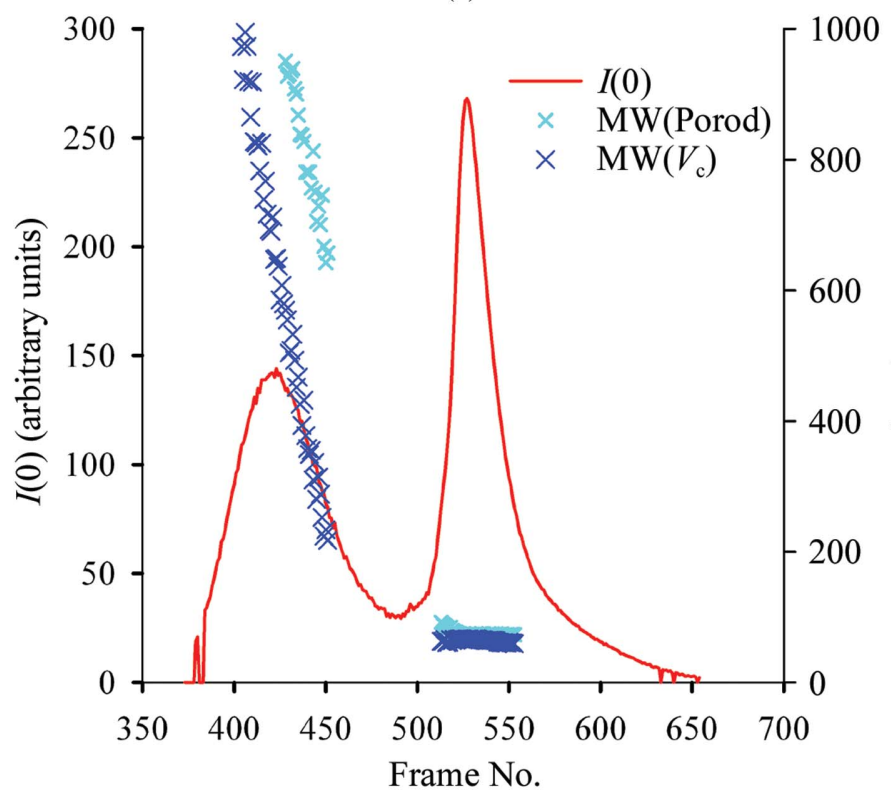

(b) samples to allow calculation of the MW and folding state for each sample frame where $R_{\mathrm{g}}$ can be estimated. Separate folder(s), named 'Peak_*', within the results directory contain frames corresponding to the detected peak(s). For each detected peak two files are created. One file contains an average over all frames in the peak and the second contains an average over $\pm 10 \%$ of all frames in the peak with reference to the maximal $I(0)$ value.

\section{Examples of DATASW usage}

Here, we describe three examples of samples with different oligomerization behaviour showing: (i) a single peak profile, (ii) multiple peaks and (iii) a profile corresponding to progressive oligomerization. Sample preparation is described in the Supporting Information. The first example describes human small heat-shock protein B6 (HSPB6; Weeks et al., 2014; Heirbaut et al., 2014). HPLC-SAXS data recorded on the SWING beamline show a single, almost symmetrical peak for HSPB6 dimers. Notably, the $R_{\mathrm{g}}$ has a slightly bell-shaped profile across the peak (Fig. 1), suggesting attractive interparticle interaction, as reported previously (Weeks et al., 2014). For data averaging, we used a sliding window size of 3 to increase the SNR. The resulting SAXS curve was essentially identical to the curve reported previously (Weeks et al., 2014). MW values calculated from the Porod volume and the volume of correlation are somewhat overestimated and underestimated, respectively (Fig. 1), although both are close to the expected MW of a dimer $(\sim 34 \mathrm{kDa})$.

The second example shows HPLC-SAXS data for a complex of the 14-3-3 protein with a binding partner (Sluchanko et al., in preparation) recorded on the BM29 beamline. The high concentration of the loaded sample

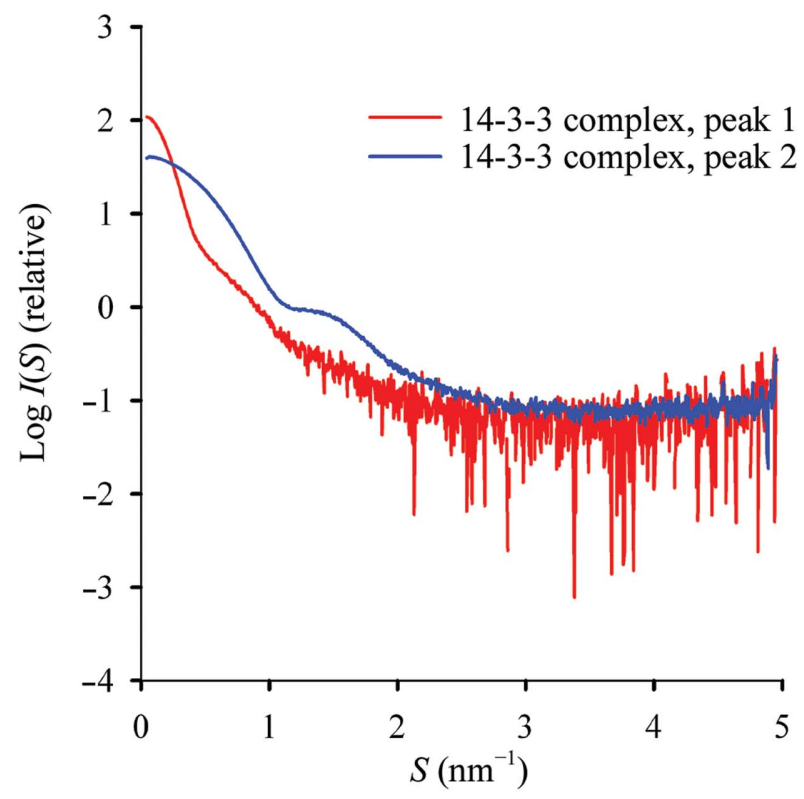

(c) 

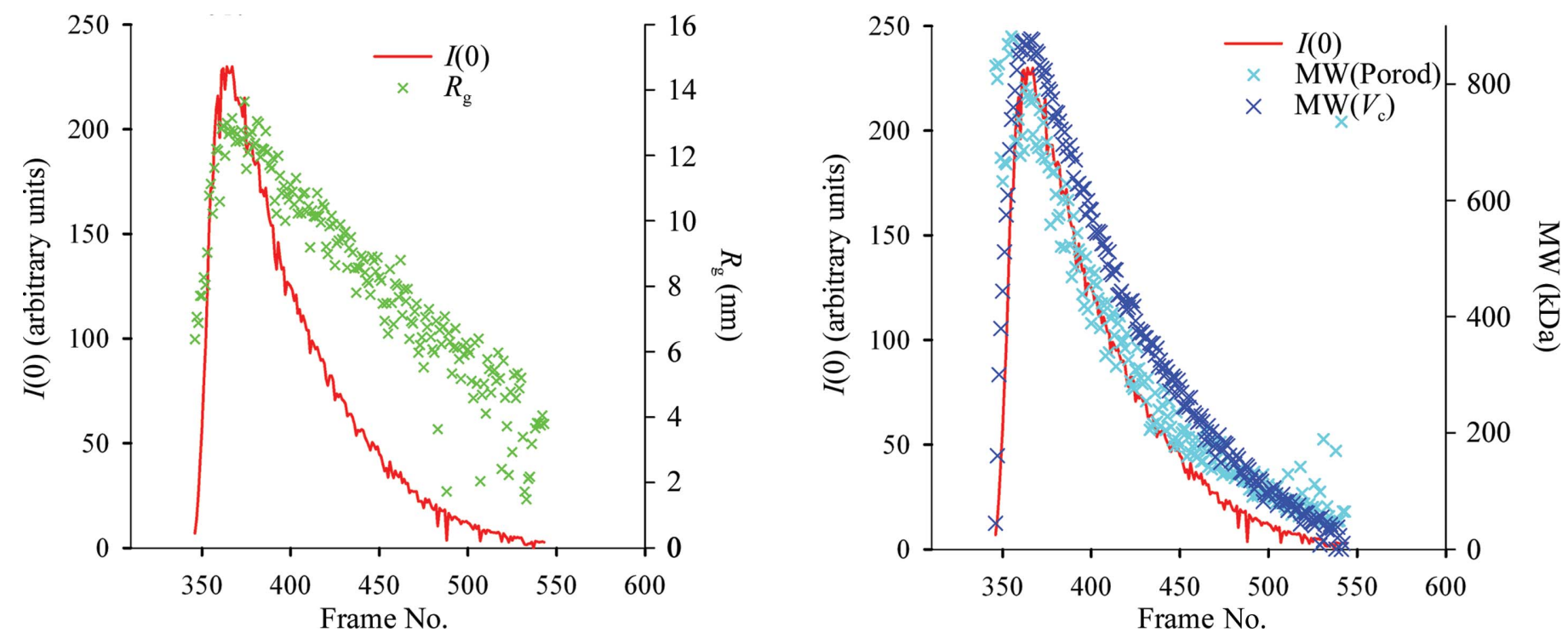

Figure 3

DATASW output for a sample (HSPB5 truncation) exhibiting progressive oligomerization. Peak detection was switched off to allow MW calculations for each sample frame.

$\left(\sim 12 \mathrm{mg} \mathrm{ml}^{-1}\right)$ and the use of the PILATUS $1 \mathrm{M}$ detector resulted in frames with a good SNR, hence additional frame averaging was not performed (sliding window size $=1$ ). The expected MW of the complex is $85 \mathrm{kDa}$. The first detected peak appears to correspond to a polydisperse oligomer with a high MW (from $\sim 1700 \mathrm{kDa}$ down to $\sim 650 \mathrm{kDa}$ ), indicated by a steep decrease in both $R_{\mathrm{g}}$ and MW with frame number (Fig. 2). The second detected peak apparently corresponds to a single species with a stable $R_{\mathrm{g}}$ value and an estimated MW of $73.5 \pm 1$ and $64.3 \pm 2 \mathrm{kDa}$ from $V_{\mathrm{p}}$ and $V_{\mathrm{c}}$, respectively (Fig. 2). A more accurate estimation of MW from the excluded volume (Supporting Information) suggests a MW of $86 \pm 1 \mathrm{kDa}$, which is in excellent agreement with the expected value.

The last example is a deletion mutant of HSPB5 (Shkumatov et al., in preparation). The SEC profile exhibited a single asymmetric peak with a long trailing right shoulder, suggesting progressive oligomerization of this sample. $D A T A S W$ was run without data-frame averaging and with peak detection disabled to perform MW estimation for every sample frame (Fig. 3). MW derived from the Porod volume decreased from $\sim 880$ to $45 \mathrm{kDa}$ (the $\mathrm{MW}$ of a monomer is $\sim 11.3 \mathrm{kDa}$ ).

In summary, DATASW is a new user-friendly tool to analyse subtracted HPLC-SAXS data and produce publication-ready plots within minutes.

\section{Acknowledgements}

We acknowledge the European Synchrotron Radiation Facility and Synchrotron SOLEIL for the provision of beam time. We would like to thank Dr M. Brennich and Dr E. Poudevigne for their help at the BM29 SAXS beamline. We are grateful to Dr A. Thureau for his help during measurements and data processing at the SWING beamline. We thank Dr N. Sluchanko, Dr Y. Sterckx and D. Guzenko for feedback and useful comments. Dr R. Sethi, Dr S. Weeks and C. Laverty are acknowledged for proofreading the manuscript. This research was supported by the KU Leuven ( $\mathrm{F}+$ fellowship to AVS and research grant OT13/097 to SVS), by the Research Foundation Flanders (FWO) grant G.0936.15 to SVS and by the European Community's Seventh Framework Programme under the BioStruct-X project.

\section{References}

Brookes, E., Demeler, B., Rosano, C. \& Rocco, M. (2010). Eur. Biophys. J. 39, 423-435.

Brookes, E., Pérez, J., Cardinali, B., Profumo, A., Vachette, P. \& Rocco, M. (2013). J. Appl. Cryst. 46, 1823-1833.

David, G. \& Pérez, J. (2009). J. Appl. Cryst. 42, 892-900.

Elegheert, J., Bracke, N., Pouliot, P., Gutsche, I., Shkumatov, A. V., Tarbouriech, N., Verstraete, K., Bekaert, A., Burmeister, W. P., Svergun, D. I., Lambrecht, B. N., Vergauwen, B. \& Savvides, S. N. (2012). Nature Struct. Mol. Biol. 19, 938-947.

Graewert, M. A. \& Svergun, D. I. (2013). Curr. Opin. Struct. Biol. 23, 748-754.

Heirbaut, M., Beelen, S., Strelkov, S. V. \& Weeks, S. D. (2014). PLoS One, 9, e105892.

Lapinaite, A., Simon, B., Skjaerven, L., Rakwalska-Bange, M., Gabel, F. \& Carlomagno, T. (2013). Nature (London), 502, 519-523.

Mathew, E., Mirza, A. \& Menhart, N. (2004). J. Synchrotron Rad. 11, 314-318.

Petoukhov, M. V., Franke, D., Shkumatov, A. V., Tria, G., Kikhney, A. G., Gajda, M., Gorba, C., Mertens, H. D. T., Konarev, P. V. \& Svergun, D. I. (2012). J. Appl. Cryst. 45, 342-350.

Rambo, R. P. \& Tainer, J. A. (2013). Nature (London), 496, 477-481. Shkumatov, A. V., Chinnathambi, S., Mandelkow, E. \& Svergun, D. I. (2011). Proteins, 79, 2122-2131.

Soderberg, C. A., Rajan, S., Shkumatov, A. V., Gakh, O., Schaefer, S., Ahlgren, E. C., Svergun, D. I., Isaya, G. \& Al-Karadaghi, S. (2013). J. Biol. Chem. 288, 8156-8167.

Weeks, S. D., Baranova, E. V., Heirbaut, M., Beelen, S., Shkumatov, A. V., Gusev, N. B. \& Strelkov, S. V. (2014). J. Struct. Biol. 185, 342-354. 